\title{
An interior penalty method for a two dimensional curl-curl and grad-div problem
}

\author{
S. C. Brenner ${ }^{1} \quad$ J. Cui ${ }^{2} \quad$ L.-Y. Sung ${ }^{3}$
}

(Received 30 October 2008; revised 30 April 2009)

\begin{abstract}
We study an interior penalty method for a two dimensional curlcurl and grad-div problem that appears in electromagnetics and in fluid-structure interactions. The method uses discontinuous $\mathrm{P}_{1}$ vector fields on graded meshes and satisfies optimal convergence rates (up to an arbitrarily small parameter) in both the energy norm and the $\mathrm{L}_{2}$ norm. These theoretical results are corroborated by results of numerical experiments.
\end{abstract}

\section{Contents}

1 Introduction

C948

2 Preliminaries

C949

3 The interior penalty method

C951

http://anziamj.austms.org.au/ojs/index.php/ANZIAMJ/article/view/1600 gives this article, (C) Austral. Mathematical Soc. 2009. Published July 1, 2009. ISSN 1446-8735. (Print two pages per sheet of paper.) 
4 Error analysis

C955

5 Nonconforming meshes

C965

6 Numerical experiments

C967

7 Concluding remarks

C970

References

C972

\section{Introduction}

Let $\Omega \subset \mathbb{R}^{2}$ be a bounded polygonal domain. We consider an interior penalty method for the following curl-curl and grad-div problem.

Problem 1 Find $\mathbf{u} \in \mathrm{H}_{0}(\operatorname{curl} ; \Omega) \cap \mathrm{H}(\operatorname{div} ; \Omega)$ such that

$$
(\nabla \times \mathbf{u}, \nabla \times \boldsymbol{v})+\gamma(\nabla \cdot \mathbf{u}, \nabla \cdot \boldsymbol{v})+\alpha(\mathbf{u}, \boldsymbol{v})=(\mathbf{f}, \boldsymbol{v})
$$

for all $\boldsymbol{v} \in \mathrm{H}_{0}(\operatorname{curl} ; \Omega) \cap \mathrm{H}(\operatorname{div} ; \Omega)$, where $(\cdot, \cdot)$ denotes the inner product of $\left[\mathrm{L}_{2}(\Omega)\right]^{2}, \alpha \in \mathbb{R}$ and $\gamma>0$ are constants, and $\mathbf{f} \in\left[\mathrm{L}_{2}(\Omega)\right]^{2}$.

The variational Problem 1 appears in electromagnetics [29, 30] and fluidstructure interactions $[27,9,8,10]$ (after interchanging the roles of curl and div). The main difficulty in the numerical solution of (1) is that standard $\mathrm{H}^{1}$-conforming finite element methods fail when $\Omega$ is not convex [22]. Such methods produce numerical solutions that converge to a vector field that is not the solution of (1). Special treatments are therefore necessary for capturing the correct solution, either by augmenting standard $\mathrm{H}^{1}$ finite element vector fields by singular vector fields $[11,5,28,3,4]$, or by solving a regularized version of (1) [24, 25, 21].

Brenner et al. [15] introduced a nonconforming finite element method for (1). It uses the Crouzeix-Raviart weakly continuous $\mathbf{P}_{1}$ vector fields [26] on graded 
meshes and has optimal convergence rates in both the energy norm and the $\mathrm{L}_{2}$ norm. In this article we study an interior penalty version of the method by Brenner et al. [15]. By removing the weak continuity condition of the vector fields, the interior penalty method applies to meshes with hanging nodes. This method belongs to a growing family of finite element methods for problems posed on $\mathrm{H}(\operatorname{curl} ; \Omega) \cap \mathrm{H}(\operatorname{div} ; \Omega)[16,15,17,20,18]$.

Section 2 recalls definitions of function spaces and properties of Problem 1, and Section 3 defines the numerical scheme whose analysis is then carried out in Section 4. Section 5 discusses the extension of the method to nonconforming meshes and Section 6 presents numerical results that corroborate the theoretical results. We end with some concluding remarks in Section 7.

\section{Preliminaries}

The function spaces $\mathrm{H}_{0}(\operatorname{curl} ; \Omega)$ and $\mathrm{H}(\operatorname{div} ; \Omega)$ are defined as

$$
\begin{aligned}
\mathrm{H}(\operatorname{curl} ; \Omega) & =\left\{\boldsymbol{v}=\left[\begin{array}{l}
v_{1} \\
v_{2}
\end{array}\right] \in\left[\mathrm{L}_{2}(\Omega)\right]^{2}: \nabla \times \boldsymbol{v}=\frac{\partial v_{2}}{\partial \mathrm{x}_{1}}-\frac{\partial v_{1}}{\partial \mathrm{x}_{2}} \in \mathrm{L}_{2}(\Omega)\right\}, \\
\mathrm{H}_{0}(\operatorname{curl} ; \Omega) & =\{\boldsymbol{v} \in \mathrm{H}(\operatorname{curl} ; \Omega): \mathfrak{n} \times \boldsymbol{v}=0 \text { on } \partial \Omega\},
\end{aligned}
$$

with $\mathfrak{n}$ being the unit outer normal, and

$$
\mathrm{H}(\operatorname{div} ; \Omega)=\left\{\boldsymbol{v}=\left[\begin{array}{l}
v_{1} \\
v_{2}
\end{array}\right] \in\left[\mathrm{L}_{2}(\Omega)\right]^{2}: \nabla \cdot \boldsymbol{v}=\frac{\partial v_{1}}{\partial x_{1}}+\frac{\partial v_{2}}{\partial x_{2}} \in \mathrm{L}_{2}(\Omega)\right\}
$$

The unique solvability of problem (1), in the case where $\alpha>0$, is guaranteed by the Riesz representation theorem for the Hilbert space $\mathrm{H}_{0}(\operatorname{curl} ; \Omega) \cap$ $\mathrm{H}(\operatorname{div} ; \Omega)$ with the inner product $((\cdot, \cdot))$ defined by

$$
((\boldsymbol{v}, \boldsymbol{w}))=(\nabla \times \boldsymbol{v}, \nabla \times \boldsymbol{w})+(\nabla \cdot \boldsymbol{v}, \nabla \cdot \boldsymbol{w})+(\boldsymbol{v}, \boldsymbol{w}) .
$$


For $\alpha \leqslant 0$, problem (1) is uniquely solvable if $\alpha$ is different from a sequence of exceptional values [30]. Indeed there exists a sequence of nonnegative numbers $0 \leqslant \lambda_{\gamma, 1} \leqslant \lambda_{\gamma, 2} \leqslant \cdots \rightarrow \infty$ such that there exists a nontrivial solution $\boldsymbol{w} \in \mathrm{H}_{0}(\operatorname{curl} ; \Omega) \cap \mathrm{H}(\operatorname{div} ; \Omega)$ for the eigenproblem

$$
(\nabla \times \boldsymbol{w}, \nabla \times \boldsymbol{v})+\gamma(\nabla \cdot \boldsymbol{w}, \nabla \cdot \boldsymbol{v})=\lambda_{\gamma, j}(\boldsymbol{w}, \boldsymbol{v})
$$

for all $\boldsymbol{v} \in \mathrm{H}_{0}(\operatorname{curl} ; \Omega) \cap \mathrm{H}(\operatorname{div} ; \Omega)$. For $\alpha \leqslant 0$, problem (1) is well-posed as long as $\alpha \neq-\lambda_{\gamma, j}$ for $j \geqslant 1$.

The regularity of the solution $\mathbf{u}$ of (1) is well established [6, 23, 15, e.g.]. Below we summarize the results as stated by Brenner et al. [15].

First of all, $\nabla \times \mathbf{u}$ and $\nabla \cdot \mathbf{u}$ belong to $\mathrm{H}^{1}(\Omega)$, and

$$
\|\nabla \times \mathbf{u}\|_{\mathrm{H}^{1}(\Omega)}+\|\nabla \cdot \mathbf{u}\|_{\mathrm{H}^{1}(\Omega)} \leqslant \mathrm{C}\|\mathbf{f}\|_{\mathrm{L}_{2}(\Omega)} .
$$

Secondly, we have $\mathbf{u} \in\left[\mathrm{H}^{2}\left(\Omega_{\delta}\right)\right]^{2}$ and the following estimate is valid:

$$
\|\mathbf{u}\|_{\mathrm{H}^{2}\left(\Omega_{\delta}\right)} \leqslant \mathrm{C}\|\mathbf{f}\|_{\mathrm{L}_{2}(\Omega)}
$$

where the domain $\Omega_{\delta}$ is obtained from $\Omega$ by excising $\delta$-neighborhoods from the corners $c_{1}, \ldots, c_{L}$ of $\Omega$, that is,

$$
\Omega_{\delta}=\left\{x \in \Omega:\left|x-c_{\ell}\right|>\delta \text { for } 1 \leqslant \ell \leqslant L\right\} .
$$

Thirdly, in the neighborhood $\mathcal{N}_{\ell, 3 \delta / 2}=\left\{x \in \Omega:\left|x-c_{\ell}\right|<3 \delta / 2\right\}$ of the corner $\mathrm{c}_{\ell}$, we have

$$
\mathbf{u}=\mathbf{u}_{\mathrm{R}}+\mathbf{u}_{\mathrm{S}}
$$

where $\boldsymbol{u}_{\mathrm{R}} \in\left[\mathrm{H}^{2-\epsilon}\left(\mathcal{N}_{\ell, 3 \delta / 2}\right)\right]^{2}$ for any $\epsilon>0$,

$$
\mathbf{u}_{\mathrm{S}}=\sum_{\substack{j \in \mathbb{N} \\
j\left(\pi / w_{\ell}\right) \in(0,2) \backslash\{1\}}} v_{\ell, j} \mathrm{j}_{\ell}^{\mathrm{j}\left(\pi / \omega_{\ell}\right)-1}\left[\begin{array}{l}
\sin \left(j\left(\pi / \omega_{\ell}\right)-1\right) \theta_{\ell} \\
\cos \left(\mathfrak{j}\left(\pi / \omega_{\ell}\right)-1\right) \theta_{\ell}
\end{array}\right],
$$


and $v_{\ell, j}$ are constants. Moreover, we have the following corner regularity estimates:

$$
\begin{aligned}
& \sum_{\ell=1}^{\mathrm{L}}\left\|\mathbf{u}_{\mathrm{R}}\right\|_{\mathrm{H}^{2-\epsilon}\left(\mathcal{N}_{\ell, 3 \delta / 2}\right)} \leqslant \mathrm{C}_{\epsilon}\|\mathbf{f}\|_{\mathrm{L}_{2}(\Omega)} ; \\
& \sum_{\ell=1}^{\mathrm{L}} \sum_{\substack{j \in \mathbb{N} \\
j\left(\pi / \boldsymbol{w}_{\ell}\right) \in(0,2) \backslash\{1\}}}\left|v_{\ell, j}\right| \leqslant \mathrm{C}\|\mathbf{f}\|_{\mathrm{L}_{2}(\Omega)} .
\end{aligned}
$$

Note that the regularity of $\nabla \times \mathbf{u}$ and $\nabla \cdot \mathbf{u}$ imply that the boundary value problem corresponding to (1) is

$$
\begin{array}{rlrl}
\nabla \times(\nabla \times \mathbf{u})-\gamma \nabla(\nabla \cdot \mathbf{u})+\alpha \mathbf{u} & =\mathbf{f} & & \text { in } \Omega, \\
\mathfrak{n} \times \mathbf{u}=0 & & \text { on } \partial \Omega, \\
\nabla \cdot \mathbf{u}=0 & & \text { on } \partial \Omega .
\end{array}
$$

\section{The interior penalty method}

We need graded meshes to recover optimal convergence rates for a general polygonal domain $\Omega$. We assume therefore that the triangulation $\mathcal{T}_{h}$ of $\Omega$ satisfies

$$
C_{1} h_{T} \leqslant h \Phi_{\mu}(T) \leqslant C_{2} h_{T} \quad \text { for all } T \in \mathcal{T}_{h},
$$

where $h_{T}$ is the diameter of the triangle $T, h=\max _{T \in \mathcal{T}_{h}} h_{T}$ is the mesh parameter, and the positive constants $C_{1}$ and $C_{2}$ are independent of $h$. The weight $\Phi_{\mu}(\mathbf{T})$ in (9) is defined by

$$
\Phi_{\mu}(\mathrm{T})=\prod_{\ell=1}^{\mathrm{L}}\left|\mathrm{c}_{\ell}-\mathrm{c}_{\mathrm{T}}\right|^{1-\mu_{\ell}},
$$


where $c_{T}$ is the center of $T$, and the grading parameters $\mu_{1}, \ldots, \mu_{\mathrm{L}}$ are chosen according to the following rule:

$$
\begin{cases}\mu_{\ell}=1 & \text { if } \omega_{\ell} \leqslant \frac{\pi}{2} \\ \mu_{\ell}<\frac{\pi}{2 \omega_{\ell}} & \text { if } \omega_{\ell}>\frac{\pi}{2}\end{cases}
$$

The construction of $\mathcal{T}_{\boldsymbol{h}}$ satisfying the mesh condition (9) is described elsewhere $\left[1,2,14,7\right.$, e.g.]. Note that $\mathcal{T}_{h}$ satisfies the minimum angle condition for any given grading parameters.

Remark 2 The choice of grading parameter in (11), which is dictated by the regularity of the solution $\mathbf{u}$ of (1), indicates that grading is needed around any corner whose angle is larger than a right angle. This is different from the grading strategy for the Laplace operator, where grading is needed only around re-entrant corners, and it is due to the singularity of the differential operator in (8a) being one order more severe than the singularity of the Laplace operator.

We take $V_{h}$ to be the space of (discontinuous) $P_{1}$ vector fields, that is,

$$
V_{h}=\left\{\boldsymbol{v} \in\left[\mathrm{L}_{2}(\Omega)\right]^{2}: \boldsymbol{v}_{\mathrm{T}}=\left.\boldsymbol{v}\right|_{\mathrm{T}} \in\left[\mathrm{P}_{1}(\mathrm{~T})\right]^{2} \quad \text { for all } \mathrm{T} \in \mathcal{T}_{\mathrm{h}}\right\} .
$$

Since the vector fields in $V_{h}$ are (in general) discontinuous, their jumps across the edges of $\mathcal{T}_{h}$ play an important role in interior penalty methods. Below are the definitions of the tangential and normal jumps of the vector fields.

We denote by $\mathcal{E}_{h}$ (respectively $\mathcal{E}_{h}^{i}$ ) the set of the edges (respectively interior edges) of $\mathcal{T}_{h}$. Let $e \in \mathcal{E}_{h}^{i}$ be shared by the two triangles $T_{ \pm} \in \mathcal{T}_{h}$ (compare with Figure 1) and $\boldsymbol{n}_{+}$(respectively $\boldsymbol{n}_{-}$) be the unit normal of $\boldsymbol{e}$ pointing towards the outside of $\mathrm{T}_{+}$(respectively $\mathrm{T}_{-}$). We define, on $\boldsymbol{e}$,

$$
\begin{aligned}
\llbracket \mathbf{n} \times \boldsymbol{v} \rrbracket & =\mathbf{n}_{+} \times\left.\boldsymbol{v}_{\mathrm{T}_{+}}\right|_{e}+\mathbf{n}_{-} \times\left.\boldsymbol{v}_{\mathrm{T}_{-}}\right|_{e}, \\
\llbracket \mathbf{n} \cdot \boldsymbol{v} \rrbracket & =\left.\mathbf{n}_{+} \cdot \boldsymbol{v}_{\mathrm{T}_{+}}\right|_{e}+\left.\mathbf{n}_{-} \cdot \boldsymbol{v}_{\mathrm{T}_{-}}\right|_{e} .
\end{aligned}
$$




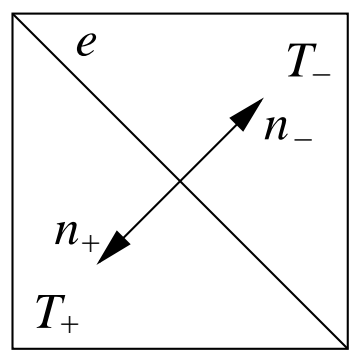

Figure 1: Triangles and normals in the definitions of $\llbracket \mathfrak{n} \times \boldsymbol{v} \rrbracket$ and $\llbracket \mathfrak{n} \cdot \boldsymbol{v} \rrbracket$.

For an edge $e \in \mathcal{E}_{\mathrm{h}}^{\mathrm{b}}$, we take $\boldsymbol{n}_{e}$ to be the unit normal of $e$ pointing towards the outside of $\Omega$ and define

$$
\llbracket \mathfrak{n} \times \boldsymbol{v} \rrbracket=\mathfrak{n}_{e} \times\left.\boldsymbol{v}\right|_{e} .
$$

We now define the discrete problem for the interior penalty method.

Problem 3 Find $\mathbf{u}_{\mathrm{h}} \in \mathrm{V}_{\mathrm{h}}$ such that

$$
\mathrm{a}_{\mathrm{h}}\left(\boldsymbol{u}_{\mathrm{h}}, \boldsymbol{v}\right)=(\mathbf{f}, \boldsymbol{v}) \quad \text { for all } \boldsymbol{v} \in \mathrm{V}_{\mathrm{h}},
$$

where

$$
\begin{aligned}
& \mathrm{a}_{\mathrm{h}}(\boldsymbol{w}, \boldsymbol{v})=\left(\nabla_{\mathrm{h}} \times \boldsymbol{w}, \nabla_{\mathrm{h}} \times \boldsymbol{v}\right)+\gamma\left(\nabla_{\mathrm{h}} \cdot \boldsymbol{w}, \nabla_{\mathrm{h}} \cdot \boldsymbol{v}\right)+\alpha(\boldsymbol{w}, \boldsymbol{v}) \\
& +\sum_{e \in \mathcal{E}_{h}} \frac{\left[\Phi_{\mu}(e)\right]^{2}}{|e|} \int_{e} \llbracket \mathfrak{n} \times \boldsymbol{w} \rrbracket \llbracket \mathfrak{n} \times \boldsymbol{v} \rrbracket d s \\
& +\sum_{e \in \mathcal{E}_{h}^{i}} \frac{\left[\Phi_{\mu}(e)\right]^{2}}{|e|} \int_{e} \llbracket \mathfrak{n} \cdot \boldsymbol{w} \rrbracket \llbracket \mathfrak{n} \cdot \boldsymbol{v} \rrbracket d s \\
& +h^{-2} \sum_{e \in \mathcal{E}_{h}} \frac{1}{|e|} \int_{e}\left(\Pi_{e}^{0} \llbracket \mathbf{n} \times \boldsymbol{w} \rrbracket\right)\left(\Pi_{e}^{0} \llbracket \mathbf{n} \times \boldsymbol{v} \rrbracket\right) d s \\
& +h^{-2} \sum_{e \in \varepsilon_{h}^{i}} \frac{1}{|e|} \int_{e}\left(\Pi_{e}^{0} \llbracket \mathfrak{n} \cdot \boldsymbol{w} \rrbracket\right)\left(\Pi_{e}^{0} \llbracket \mathfrak{n} \cdot \boldsymbol{v} \rrbracket\right) d s,
\end{aligned}
$$


and where $|\mathrm{e}|$ denotes the length of the edge $\mathrm{e}$, and $\Pi_{\mathrm{e}}^{0}$ is the orthogonal projection from $\mathrm{L}_{2}(\mathrm{e})$ to $\mathrm{P}_{0}(\mathrm{e})$ (the space of constant functions on $\mathrm{e}$ ). The edge weight $\Phi_{\mu}(e)$ in (15) is defined by

$$
\Phi_{\mu}(e)=\prod_{\ell=1}^{\mathrm{L}}\left|\mathrm{c}_{\ell}-\mathrm{m}_{e}\right|^{1-\mu_{\ell}},
$$

where $\mathrm{c}_{1}, \ldots, \mathrm{c}_{\mathrm{L}}$ are the corners of $\Omega$ and $\mathrm{m}_{e}$ is the midpoint of the edge e. Remark 4 Comparing (10) and (16) we have

$$
\Phi_{\mu}(e) \approx \Phi_{\mu}(T) \text { if } e \subset \partial T,
$$

where the positive constants in the equivalence are independent of $h$. This relation is important for the derivation of optimal a priori error estimates.

We use the Crouzeix-Raviart interpolation operator in the analysis of the interior penalty method. For $s>1 / 2$ and $T \in \mathcal{T}_{h}$, we define $\Pi_{\mathrm{T}}:\left[\mathrm{H}^{\mathrm{s}}(\mathrm{T})\right]^{2} \rightarrow$ $\left[\mathrm{P}_{1}(\mathrm{~T})\right]^{2}$ by

$$
\int_{e_{j}}\left(\Pi_{T} \zeta\right) d s=\int_{e_{j}} \zeta d s \quad \text { for } 1 \leqslant j \leqslant 3
$$

where $e_{1}, e_{2}$ and $e_{3}$ are the edges of $T$. The operator $\Pi_{\mathrm{T}}$ satisfies the following standard error estimate [26]:

$$
\left\|\zeta-\Pi_{\mathrm{T}} \zeta\right\|_{\mathrm{L}_{2}(\mathrm{~T})}+\mathrm{h}_{\mathrm{T}}^{\min (s, 1)}\left|\zeta-\Pi_{\mathrm{T}} \zeta\right|_{\mathrm{H}^{\min (s, 1)}(\mathrm{T})} \leqslant \mathrm{C}_{\mathrm{T}} \mathrm{h}_{\mathrm{T}}^{\mathrm{s}}|\zeta|_{\mathrm{H}^{\mathrm{s}}(\mathrm{T})}
$$

for all $\zeta \in\left[H^{s}(T)\right]^{2}$ and $s \in(1 / 2,2]$, where the positive constant $C_{T}$ depends on the minimum angle of $T$ (and also on $s$ when $s$ approaches $1 / 2$ ).

Since $H_{0}(\operatorname{curl} ; \Omega) \cap H(\operatorname{div} ; \Omega) \subset\left[H^{s}(\Omega)\right]^{2}$ for some $s>1 / 2$ [30, 15, cf. e.g.], we can define a global interpolation operator

$$
\Pi_{\mathrm{h}}: \mathrm{H}_{\mathrm{o}}(\operatorname{curl} ; \Omega) \cap \mathrm{H}(\operatorname{div} ; \Omega) \rightarrow \mathrm{V}_{\mathrm{h}}
$$

by piecing together the local interpolation operators, that is,

$$
\left(\Pi_{\mathrm{h}} \boldsymbol{v}\right)_{\mathrm{T}}=\Pi_{\mathrm{T}} \boldsymbol{v}_{\mathrm{T}} \quad \text { for all } \mathrm{T} \in \mathcal{T}_{\mathrm{h}} .
$$


We also denote the piecewise defined curl and div operator by $\nabla_{h} \times$ and $\nabla_{h}$, that is,

$$
\begin{aligned}
\left(\nabla_{\mathrm{h}} \times \boldsymbol{v}\right)_{\mathrm{T}} & =\nabla \times\left(\boldsymbol{v}_{\mathrm{T}}\right) \quad \text { for all } \mathrm{T} \in \mathcal{T}_{\mathrm{h}}, \\
\left(\nabla_{\mathrm{h}} \cdot \boldsymbol{v}\right)_{\mathrm{T}} & =\nabla \cdot\left(\boldsymbol{v}_{\mathrm{T}}\right) \quad \text { for all } \mathrm{T} \in \mathcal{T}_{\mathrm{h}} .
\end{aligned}
$$

It follows from (18), (20)-(22) and Green's theorem that

$$
\begin{array}{cc}
\nabla_{\mathrm{h}} \times\left(\Pi_{\mathrm{h}} \boldsymbol{v}\right)=\Pi_{0}^{\mathrm{h}}(\nabla \times \boldsymbol{v}) & \text { for all } \boldsymbol{v} \in \mathrm{H}_{\mathrm{o}}(\operatorname{curl} ; \Omega) \cap \mathrm{H}(\operatorname{div} ; \Omega), \\
\nabla_{\mathrm{h}} \cdot\left(\Pi_{\mathrm{h}} \boldsymbol{v}\right)=\Pi_{0}^{\mathrm{h}}(\nabla \cdot \boldsymbol{v}) & \text { for all } \boldsymbol{v} \in \mathrm{H}_{\mathrm{o}}(\operatorname{curl} ; \Omega) \cap \mathrm{H}(\operatorname{div} ; \Omega),
\end{array}
$$

where $\Pi_{0}^{h}$ is the orthogonal projection from $L_{2}(\Omega)$ onto the space of piecewise constant functions associated with $\mathcal{T}_{h}$.

\section{Error analysis}

The discretization error is measured in both the $\mathrm{L}_{2}$ norm and the mesh dependent energy norm $\|\cdot\|_{h}$ defined by

$$
\begin{aligned}
\|\boldsymbol{v}\|_{\mathrm{h}}^{2}= & \left\|\nabla_{\mathrm{h}} \times \boldsymbol{v}\right\|_{\mathrm{L}_{2}(\Omega)}^{2}+\gamma\left\|\nabla_{\mathrm{h}} \cdot \boldsymbol{v}\right\|_{\mathrm{L}_{2}(\Omega)}^{2}+\|\boldsymbol{v}\|_{\mathrm{L}_{2}(\Omega)}^{2} \\
& +\sum_{e \in \mathcal{E}_{\mathrm{h}}} \frac{\left[\Phi_{\mu}(e)\right]^{2}}{|\boldsymbol{e}|}\|\llbracket \mathfrak{n} \times \boldsymbol{v} \rrbracket\|_{\mathrm{L}_{2}(e)}^{2}+\sum_{e \in \mathcal{E}_{\mathrm{h}}^{i}} \frac{\left[\Phi_{\mu}(e)\right]^{2}}{|\boldsymbol{e}|}\|\llbracket \mathfrak{n} \cdot \boldsymbol{v} \rrbracket\|_{\mathrm{L}_{2}(e)}^{2} \\
& +\mathrm{h}^{-2}\left(\sum_{e \in \mathcal{E}_{h}} \frac{1}{|\boldsymbol{e}|}\left\|\Pi_{\mathrm{e}}^{0} \llbracket \mathfrak{n} \times \boldsymbol{v} \rrbracket\right\|_{\mathrm{L}_{2}(e)}^{2}+\sum_{e \in \mathcal{E}_{\mathrm{h}}^{i}} \frac{1}{|\boldsymbol{e}|}\left\|\Pi_{e}^{0} \llbracket \mathfrak{n} \cdot \boldsymbol{v} \rrbracket\right\|_{\mathrm{L}_{2}(e)}^{2}\right) .
\end{aligned}
$$

Note that $a_{h}(\cdot, \cdot)$ is bounded by this energy norm, that is,

$$
\left|a_{h}(\boldsymbol{w}, v)\right| \leqslant(|\alpha|+1)\|\boldsymbol{w}\|_{h}\|v\|_{h}
$$

for all $\boldsymbol{v}, \boldsymbol{w} \in \mathrm{H}_{\mathrm{o}}(\operatorname{curl} ; \Omega) \cap \mathrm{H}(\operatorname{div} ; \Omega)+\mathrm{V}_{\mathrm{h}}$. 
For $\alpha>0, a_{h}(\cdot, \cdot)$ is also coercive with respect to $\|\cdot\|_{h}$, that is,

$$
a_{h}(v, v) \geqslant \min (1, \alpha)\|v\|_{h}^{2}
$$

for all $\boldsymbol{v} \in \mathrm{H}_{0}(\operatorname{curl} ; \Omega) \cap \mathrm{H}(\operatorname{div} ; \Omega)+\mathrm{V}_{\mathrm{h}}$. In this case the discrete problem is well-posed and we have the following abstract error estimate, whose proof is identical with our earlier proof [18, Lemma 3.5].

Lemma 5 Let $\alpha$ be positive, $\beta=\min (1, \alpha), \mathbf{u} \in \mathrm{H}_{0}(\operatorname{curl} ; \Omega) \cap \mathrm{H}(\operatorname{div} ; \Omega)$ be the solution of (1), and $\mathbf{u}_{\mathrm{h}}$ satisfy the discrete problem (14). Then

$$
\left\|\mathbf{u}-\mathbf{u}_{\mathrm{h}}\right\|_{h} \leqslant\left(\frac{1+\alpha+\beta}{\beta}\right) \inf _{\boldsymbol{v} \in V_{h}}\|\mathbf{u}-\boldsymbol{v}\|_{h}+\frac{1}{\beta} \sup _{\boldsymbol{w} \in V_{h} \backslash\{0\}} \frac{a_{h}\left(\mathbf{u}-\mathbf{u}_{h}, \boldsymbol{w}\right)}{\|\boldsymbol{w}\|_{h}} .
$$

For $\alpha \leqslant 0$, the following Gårding (in)equality holds

$$
a_{h}(v, v)+(|\alpha|+1)(v, v)=\|v\|_{h}^{2}
$$

for all $\boldsymbol{v} \in \mathrm{H}_{0}(\operatorname{curl} ; \Omega) \cap \mathrm{H}(\operatorname{div} ; \Omega)+\mathrm{V}_{\mathrm{h}}$. In this case the discrete problem is indefinite and the following lemma provides an abstract error estimate for the scheme (14) under the assumption that it has a solution. Its proof, which is based on (26) and (29), is also identical to our earlier proof [18, Lemma 3.6].

Lemma 6 Let $\mathbf{u} \in \mathrm{H}_{\mathrm{o}}(\operatorname{curl} ; \Omega) \cap \mathrm{H}(\operatorname{div} ; \Omega)$ satisfy (1) and $\mathbf{u}_{\mathrm{h}}$ be a solution of (14). Then

$$
\begin{aligned}
\left\|\mathbf{u}-\mathbf{u}_{\mathrm{h}}\right\|_{\mathrm{h}} \leqslant & (2|\alpha|+3) \inf _{\boldsymbol{v} \in \mathrm{V}_{\mathrm{h}}}\|\mathbf{u}-\boldsymbol{v}\|_{\mathrm{h}}+\sup _{\boldsymbol{w} \in V_{\mathrm{h}} \backslash\{0\}} \frac{\boldsymbol{a}_{\mathrm{h}}\left(\mathbf{u}-\mathbf{u}_{\mathrm{h}}, \boldsymbol{w}\right)}{\|\boldsymbol{w}\|_{\mathrm{h}}} \\
& +(|\alpha|+1)\left\|\mathbf{u}-\mathbf{u}_{\mathrm{h}}\right\|_{\mathrm{L}_{2}(\Omega)} .
\end{aligned}
$$

From here on we consider $\alpha$ and $\gamma$ to be fixed and drop the dependence on these constants in our estimates. 
Remark 7 The first term on the right-hand side of (28) and (30) measures the approximation property of $V_{h}$ with respect to the energy norm. The second term measures the consistency error. The third term on the righthand side of (30) addresses the indefiniteness of the problem when $\alpha \leqslant 0$.

Since the interpolation operator $\Pi_{h}$ defined in Section 3 is also the one employed in earlier work [16], we use in our analysis the following results from that article [16, Lemma 5.1 and Lemma 5.2] which were obtained by using (11), (17), (19) and the regularity estimates (3)-(7).

Lemma 8 Let $\mathbf{u} \in \mathrm{H}_{0}(\operatorname{curl} ; \Omega) \cap \mathrm{H}(\operatorname{div} ; \Omega)$ be the solution of $(1)$. We have the following interpolation error estimates:

$$
\begin{aligned}
& \left\|\mathbf{u}-\Pi_{\mathrm{h}} \mathbf{u}\right\|_{\mathrm{L}_{2}(\Omega)} \leqslant \mathrm{h}^{2-\epsilon}\|\mathbf{f}\|_{\mathrm{L}_{2}(\Omega)}, \\
& \sum_{e \in \mathcal{E}_{h}} \frac{\left[\Phi_{\mu}(e)\right]^{2}}{|e|}\left\|\llbracket \mathbf{u}-\Pi_{h} \mathbf{u} \rrbracket\right\|_{\mathrm{L}_{2}(e)}^{2} \leqslant C_{\epsilon} h^{2-\epsilon}\|\mathbf{f}\|_{\mathrm{L}_{2}(\Omega)}^{2},
\end{aligned}
$$

for any $\boldsymbol{\epsilon}>0$, where $\llbracket \mathbf{u}-\Pi_{\mathrm{h}} \mathbf{u} \rrbracket$ is the jump of $\mathbf{u}-\Pi_{\mathrm{h}} \mathbf{u}$ across the interior edges of $\mathcal{T}_{h}$ and $\llbracket \mathbf{u}-\Pi_{h} \mathbf{u} \rrbracket$ is $\mathbf{u}-\Pi_{h} \mathbf{u}$ on the boundary edges of $\mathcal{T}_{h}$.

The approximation property of $V_{h}$ is established by the following lemma.

Lemma 9 Let $\mathbf{u} \in \mathrm{H}_{0}(\operatorname{curl} ; \Omega) \cap \mathrm{H}(\operatorname{div} ; \Omega)$ be the solution of (1). Then

$$
\inf _{\boldsymbol{v} \in V_{h}}\|\mathbf{u}-\boldsymbol{v}\|_{h} \leqslant\left\|\mathbf{u}-\Pi_{h} \mathbf{u}\right\|_{h}<C_{\epsilon} h^{1-\epsilon}\|\mathfrak{f}\|_{L_{2}(\Omega)}
$$

for any $\epsilon>0$.

Proof: It follows from (18) that $\Pi_{e}^{0} \llbracket \mathfrak{n} \times\left(\mathbf{u}-\Pi_{h} \mathbf{u}\right) \rrbracket=0$ for all $\boldsymbol{e} \in \mathcal{E}_{\mathrm{h}}$ and $\Pi_{e}^{0} \llbracket \mathfrak{n} \cdot\left(\mathbf{u}-\Pi_{h} \mathbf{u}\right) \rrbracket=0$ for all $e \in \mathcal{E}_{h}^{i}$. Therefore we have

$$
\begin{aligned}
\left\|\mathbf{u}-\Pi_{\mathrm{h}} \mathbf{u}\right\|_{\mathrm{h}}^{2}= & \left\|\nabla_{\mathrm{h}} \times\left(\mathbf{u}-\Pi_{\mathrm{h}} \mathbf{u}\right)\right\|_{\mathrm{L}_{2}(\Omega)}^{2} \\
& +\gamma\left\|\nabla_{\mathrm{h}} \cdot\left(\mathbf{u}-\Pi_{\mathrm{h}} \mathbf{u}\right)\right\|_{\mathrm{L}_{2}(\Omega)}^{2}+\left\|\mathbf{u}-\Pi_{\mathrm{h}} \mathbf{u}\right\|_{\mathrm{L}_{2}(\Omega)}^{2}
\end{aligned}
$$




$$
\begin{aligned}
& +\sum_{e \in \mathcal{E}_{\mathrm{h}}} \frac{\left[\Phi_{\mu}(e)\right]^{2}}{|e|}\left\|\llbracket \mathfrak{n} \times\left(\mathbf{u}-\Pi_{\mathrm{h}} \mathbf{u}\right) \rrbracket\right\|_{\mathrm{L}_{2}(e)}^{2} \\
& +\sum_{e \in \mathcal{E}_{\mathrm{h}}^{i}} \frac{\left[\Phi_{\mu}(e)\right]^{2}}{|e|}\left\|\llbracket \mathfrak{n} \cdot\left(\mathbf{u}-\Pi_{\mathrm{h}} \mathbf{u}\right) \rrbracket\right\|_{\mathrm{L}_{2}(e)}^{2} .
\end{aligned}
$$

Lemma 8 estimates the last three terms on the right-hand side of (34), and we bound the first two terms on the right-hand side of (34) by (3), (19), (23) and (24) as

$$
\begin{aligned}
& \left\|\nabla_{\mathrm{h}} \times\left(\mathbf{u}-\Pi_{\mathrm{h}} \mathbf{u}\right)\right\|_{\mathrm{L}_{2}(\Omega)}^{2}+\gamma\left\|\nabla_{\mathrm{h}} \cdot\left(\mathbf{u}-\Pi_{\mathrm{h}} \mathbf{u}\right)\right\|_{\mathrm{L}_{2}(\Omega)}^{2} \\
& =\left\|\nabla \times \mathbf{u}-\Pi_{0}^{\mathrm{h}}(\nabla \times \mathbf{u})\right\|_{\mathrm{L}_{2}(\Omega)}^{2}+\gamma\left\|\nabla \cdot \mathbf{u}-\Pi_{0}^{\mathrm{h}}(\nabla \cdot \mathbf{u})\right\|_{\mathrm{L}_{2}(\Omega)}^{2} \\
& \leqslant \mathrm{Ch}^{2}\|\mathbf{f}\|_{\mathrm{L}_{2}(\Omega)}^{2} .
\end{aligned}
$$

Next we turn to the consistency error, where we need the following result proved in earlier work [16, Lemma 5.3].

\section{Lemma 10}

$$
\sum_{e \in \mathcal{E}_{\mathrm{h}}}|e|\left[\Phi_{\mu}(e)\right]^{-2}\left\|\eta-\hat{\Upsilon}_{\mathrm{T}_{e}}\right\|_{\mathrm{L}_{2}(e)}^{2} \leqslant \mathrm{Ch}^{2}|\eta|_{\mathrm{H}^{1}(\Omega)}^{2} \quad \text { for all } \eta \in \mathrm{H}^{1}(\Omega),
$$

where $\hat{\eta}_{\mathrm{T}_{e}}=\left|\mathrm{T}_{e}\right|^{-1} \int_{\mathrm{T}_{e}} \eta \mathrm{dx}$ is the mean of $\eta$ over $\mathrm{T}_{e}$, one of the triangles in $\mathcal{T}_{\mathrm{h}}$ that has $\mathrm{e}$ as an edge.

The following lemma provides an optimal bound for the consistency error.

Lemma 11 Let $\mathbf{u} \in \mathrm{H}_{0}(\operatorname{curl} ; \Omega) \cap \mathrm{H}(\operatorname{div} ; \Omega)$ be the solution of (1) and $\mathbf{u}_{\mathrm{h}} \in \mathrm{V}_{\mathrm{h}}$ satisfy (14). Then

$$
\sup _{\boldsymbol{w} \in V_{h} \backslash\{0\}} \frac{a_{h}\left(\mathbf{u}-\mathbf{u}_{h}, \boldsymbol{w}\right)}{\|\boldsymbol{w}\|_{h}} \leqslant \operatorname{Ch}\|\mathbf{f}\|_{L_{2}(\Omega)} .
$$


Proof: Let $\boldsymbol{w} \in \mathrm{V}_{\mathrm{h}}$ be arbitrary. Since the strong form of (1) is given by (8), we find, by (12), (13), (15) and integration by parts,

$$
\begin{aligned}
& \mathrm{a}_{\mathrm{h}}(\mathbf{u}, \boldsymbol{w})=\sum_{\mathrm{T} \in \mathcal{T}_{\mathrm{h}}} \int_{\mathrm{T}}(\nabla \times \mathbf{u})(\nabla \times \boldsymbol{w}) \mathrm{d} x \\
& +\sum_{\mathrm{T} \in \mathcal{T}_{\mathrm{h}}} \gamma \int_{\mathrm{T}}(\nabla \cdot \mathbf{u})(\nabla \cdot \boldsymbol{w}) \mathrm{d} x+\alpha(\mathbf{u}, \boldsymbol{w}) \\
& =(\mathbf{f}, \boldsymbol{w})+\sum_{\mathrm{e} \in \mathcal{E}_{\mathrm{h}}} \int_{e}(\nabla \times \mathbf{u}) \llbracket \mathfrak{n} \times \boldsymbol{w} \rrbracket \mathrm{d} \mathbf{s} \\
& +\sum_{\mathfrak{e} \in \mathcal{E}_{\mathrm{h}}^{\mathrm{i}}} \gamma \int_{e}(\nabla \cdot \mathfrak{u}) \llbracket \mathfrak{n} \cdot \boldsymbol{w} \rrbracket \mathrm{ds} .
\end{aligned}
$$

Subtracting (14) from (36) gives

$$
a_{h}\left(\mathbf{u}-\mathbf{u}_{\mathrm{h}}, \boldsymbol{w}\right)=\sum_{\mathrm{e} \in \mathcal{E}_{\mathrm{h}}} \int_{e}(\nabla \times \mathbf{u}) \llbracket \mathfrak{n} \times \boldsymbol{w} \rrbracket \mathrm{d} s+\sum_{e \in \mathcal{E}_{\mathrm{h}}^{i}} \gamma \int_{e}(\nabla \cdot \mathbf{u}) \llbracket \mathfrak{n} \cdot \boldsymbol{w} \rrbracket \mathrm{d} s .
$$

We rewrite the first term on the right-hand side of (37) as

$$
\begin{aligned}
\sum_{\boldsymbol{e} \in \mathcal{E}_{\mathrm{h}}} \int_{e}(\nabla \times \mathbf{u}) \llbracket \mathfrak{n} \times \boldsymbol{w} \rrbracket d s= & \sum_{e \in \mathcal{E}_{\mathrm{h}}} \int_{e}\left(\nabla \times \mathbf{u}-(\widehat{\nabla \times \mathfrak{u}})_{\mathrm{T}_{e}}\right) \llbracket \mathfrak{n} \times \boldsymbol{w} \rrbracket \mathrm{d} s \\
& +\sum_{e \in \mathcal{E}_{\mathrm{h}}} \int_{e}(\widehat{\nabla \times \mathfrak{u}})_{\mathrm{T}_{e}}\left(\Pi_{e}^{0} \llbracket \mathfrak{n} \times \boldsymbol{w} \rrbracket\right) \mathrm{ds},
\end{aligned}
$$

where $(\widehat{\nabla \times \mathfrak{u}})_{\mathrm{T}_{e}}$ is the mean of $\nabla \times \mathbf{u}$ on $\mathrm{T}_{\boldsymbol{e}}$, one of the triangles in $\mathcal{T}_{\boldsymbol{h}}$ that has $\boldsymbol{e}$ as an edge.

It follows from (3), (25), Lemma 10 and the Cauchy-Schwarz inequality that the first term on the right-hand side of (38) satisfies

$$
\sum_{\mathfrak{e} \in \mathcal{E}_{h}} \int_{e}\left(\nabla \times \mathbf{u}-(\widehat{\nabla \times \mathfrak{u}})_{\mathrm{T}_{e}}\right) \llbracket \mathfrak{n} \times \boldsymbol{w} \rrbracket \mathrm{d} s
$$




$$
\begin{aligned}
\leqslant & \left(\sum_{e \in \mathcal{E}_{h}}|e|\left[\Phi_{\mu}(e)\right]^{-2} \| \nabla \times \mathbf{u}-\left(\widehat{\nabla \times \mathfrak{u})} \|_{\mathrm{L}_{2}(e)}^{2}\right)^{1 / 2}\right. \\
& \times\left(\sum_{e \in \mathcal{E}_{h}}|e|^{-1}\left[\Phi_{\mu}(e)\right]^{2}\|\mathbf{n} \times \boldsymbol{w}\|_{\mathrm{L}_{2}(e)}^{2}\right)^{1 / 2} \\
\leqslant & \operatorname{Ch}\|\mathbf{f}\|_{\mathrm{L}_{2}(\Omega)}\|\boldsymbol{w}\|_{h} .
\end{aligned}
$$

For the second term on the right-hand side of (38), we find, by using the Cauchy-Schwarz inequality, (3) and (25),

$$
\begin{aligned}
& \sum_{e \in \mathcal{E}_{\mathrm{h}}} \int_{e}(\widehat{\nabla \times \mathfrak{u}})_{\mathrm{T}_{e}}\left(\Pi_{\mathrm{h}}^{0} \llbracket \mathfrak{n} \times \boldsymbol{w} \rrbracket\right) \mathrm{ds} \\
& \leqslant \sum_{e \in \mathcal{E}_{\mathrm{h}}}\left(|e|^{1 / 2}\left\|(\widehat{\nabla \times \mathfrak{u}})_{\mathrm{T}_{e}}\right\|_{\mathrm{L}_{2}(e)}\right)\left(|e|^{-1 / 2}\left\|\Pi_{e}^{0} \llbracket \mathfrak{n} \times \boldsymbol{w} \rrbracket\right\|_{\mathrm{L}_{2}(e)}\right) \\
& \leqslant \mathrm{Ch}\left(\sum_{e \in \mathcal{E}_{\mathrm{h}}} \|\left(\widehat{\nabla \times \mathfrak{u})_{\mathrm{T}_{e}}} \|_{\mathrm{L}_{2}\left(\mathrm{~T}_{e}\right)}^{2}\right)^{1 / 2}\right. \\
& \times\left(\mathrm{h}^{-2} \sum_{e \in \mathcal{E}_{\mathrm{h}}} \frac{1}{|\boldsymbol{e}|}\left\|\Pi_{e}^{0} \llbracket \mathfrak{n} \times \boldsymbol{w} \rrbracket\right\|_{\mathrm{L}_{2}(e)}^{2}\right)^{1 / 2} \\
& \leqslant \mathrm{Ch}\|\nabla \times \boldsymbol{u}\|_{\mathrm{L}_{2}(\Omega)}\|\boldsymbol{w}\|_{h} \\
& \leqslant \mathrm{Ch}\|\mathbf{f}\|_{\mathrm{L}_{2}(\Omega)}\|\boldsymbol{w}\|_{\mathrm{h}} \text {. }
\end{aligned}
$$

Here we have also used the fact that, if $e$ is an edge of a triangle $T$, then

$$
|e|\|q\|_{\mathrm{L}_{2}(e)}^{2} \leqslant \mathrm{C}_{\mathrm{T}}\|\mathrm{q}\|_{\mathrm{L}_{2}(\mathrm{~T})}^{2} \text { for any constant function } \mathrm{q} \text {, }
$$

where the positive constant $C_{T}$ depends only on the shape of $T$.

Combining (38)-(40), we have

$$
\sum_{\boldsymbol{e} \in \mathcal{E}_{\mathrm{h}}} \int_{e}(\nabla \times \mathfrak{u}) \llbracket \mathfrak{n} \cdot \boldsymbol{w} \rrbracket \mathrm{ds} \leqslant \mathrm{Ch}\|\mathbf{f}\|_{\mathrm{L}_{2}(\Omega)}\|\boldsymbol{w}\|_{\mathrm{h}},
$$


and similarly,

$$
\sum_{\mathfrak{e} \in \mathcal{E}_{\mathrm{h}}^{i}} \int_{e}(\nabla \cdot \mathfrak{u}) \llbracket \mathfrak{n} \cdot \boldsymbol{w} \rrbracket d s \leqslant \mathrm{Ch}\|\mathbf{f}\|_{\mathrm{L}_{2}(\Omega)}\|\boldsymbol{w}\|_{\mathrm{h}} .
$$

The estimate (35) follows from (37), (42) and (43).

The following lemma gives an $\mathrm{L}_{2}$ error estimate under the assumption that the discrete problem (14) has a solution.

Lemma 12 Let $\mathbf{u} \in \mathrm{H}_{0}(\operatorname{curl} ; \Omega) \cap \mathrm{H}(\operatorname{div} ; \Omega)$ be the solution of (1) and $\mathbf{u}_{\mathrm{h}} \in \mathrm{V}_{\mathrm{h}}$ satisfy (14). Then

$$
\left\|\mathbf{u}-\mathbf{u}_{\mathrm{h}}\right\|_{\mathrm{L}_{2}(\Omega)} \leqslant \mathrm{C}_{\epsilon}\left(\mathrm{h}^{2-\epsilon}\|\mathbf{f}\|_{\mathrm{L}_{2}(\Omega)}+\mathrm{h}^{1-\epsilon}\left\|\mathbf{u}-\mathbf{u}_{\mathrm{h}}\right\|_{\mathrm{h}}\right)
$$

for any $\epsilon>0$.

Proof: The proof is based on a duality argument. Let $z \in \mathrm{H}_{0}(\operatorname{curl} ; \Omega) \cap$ $\mathrm{H}(\operatorname{div} ; \Omega)$ satisfy

$$
(\nabla \times \boldsymbol{v}, \nabla \times \boldsymbol{z})+\gamma(\nabla \cdot \boldsymbol{v}, \nabla \cdot \boldsymbol{z})+\alpha(\boldsymbol{v}, \boldsymbol{z})=\left(\boldsymbol{v},\left(\mathbf{u}-\mathbf{u}_{\mathrm{h}}\right)\right)
$$

for all $v \in \mathrm{H}_{0}(\operatorname{curl} ; \Omega) \cap \mathrm{H}(\operatorname{div} ; \Omega)$. The strong form of (45) is

$$
\begin{aligned}
\nabla \times(\nabla \times \boldsymbol{z})-\gamma \nabla(\nabla \cdot \boldsymbol{z})+\alpha \boldsymbol{z} & =\mathbf{u}-\mathbf{u}_{\mathrm{h}} & & \text { in } \Omega, \\
\mathbf{n} \times \boldsymbol{z} & =0 & & \text { on } \partial \Omega, \\
\nabla \cdot \boldsymbol{z} & =0 & & \text { on } \partial \Omega,
\end{aligned}
$$

and we have the following analog of (3):

$$
\|\nabla \times \boldsymbol{z}\|_{H^{1}(\Omega)}+\|\nabla \cdot \boldsymbol{z}\|_{H^{1}(\Omega)} \leqslant \mathrm{C}\left\|\mathbf{u}-\mathbf{u}_{\mathrm{h}}\right\|_{\mathrm{L}_{2}(\Omega)} .
$$

Furthermore we can write (45) as

$$
a_{h}(\boldsymbol{v}, z)=\left(\boldsymbol{v},\left(\boldsymbol{u}-\mathbf{u}_{\mathrm{h}}\right)\right) \quad \text { for all } \boldsymbol{v} \in \mathrm{H}_{\mathrm{o}}(\operatorname{curl} ; \Omega) \cap \mathrm{H}(\operatorname{div} ; \Omega) .
$$


It follows from (46), (48) and integration by parts that the following analog of (36) holds:

$$
\begin{aligned}
\mathbf{a}_{\mathrm{h}}\left(\mathbf{u}_{\mathrm{h}}, \boldsymbol{z}\right)= & \sum_{\mathrm{T} \in \mathcal{T}_{\mathrm{h}}} \int_{\mathrm{T}}\left(\nabla \times \mathbf{u}_{\mathrm{h}}\right)(\nabla \times \boldsymbol{z}) \mathrm{d} x \\
& +\sum_{\mathrm{T} \in \mathcal{T}_{\mathrm{h}}} \gamma \int_{\mathrm{T}}\left(\nabla \cdot \mathbf{u}_{\mathrm{h}}\right)(\nabla \cdot \boldsymbol{z}) \mathrm{d} x+\alpha\left(\mathbf{u}_{\mathrm{h}}, \boldsymbol{z}\right) \\
= & \left(\mathbf{u}_{\mathrm{h}},\left(\mathbf{u}-\mathbf{u}_{\mathrm{h}}\right)\right)+\sum_{\boldsymbol{e} \in \mathcal{E}_{\mathrm{h}}} \int_{e} \llbracket \mathfrak{n} \times \mathbf{u}_{\mathrm{h}} \rrbracket(\nabla \times \boldsymbol{z}) \mathrm{d} s \\
& +\sum_{e \in \mathcal{E}_{\mathrm{h}}^{i}} \gamma \int_{e} \llbracket \mathfrak{n} \cdot \mathbf{u}_{\mathrm{h}} \rrbracket(\nabla \cdot \boldsymbol{z}) \mathrm{ds} .
\end{aligned}
$$

Combining (48) and (49) gives

$$
\begin{aligned}
\left\|\mathbf{u}-\mathbf{u}_{\mathrm{h}}\right\|_{\mathrm{L}_{2}(\Omega)}^{2}= & \left(\mathbf{u}, \mathbf{u}-\mathbf{u}_{\mathrm{h}}\right)-\left(\mathbf{u}_{\mathrm{h}}, \mathbf{u}-\mathbf{u}_{\mathrm{h}}\right) \\
= & a_{\mathrm{h}}\left(\mathbf{u}-\mathbf{u}_{\mathrm{h}}, \boldsymbol{z}\right)+\sum_{\boldsymbol{e} \in \mathcal{E}_{\mathrm{h}}} \int_{e} \llbracket \mathfrak{n} \times \mathbf{u}_{\mathrm{h}} \rrbracket(\nabla \times \boldsymbol{z}) \mathrm{d} s \\
& +\sum_{\mathbf{e} \in \mathcal{E}_{\mathrm{h}}^{i}} \gamma \int_{e} \llbracket \mathfrak{n} \cdot \mathbf{u}_{\mathrm{h}} \rrbracket(\nabla \cdot \boldsymbol{z}) \mathrm{ds},
\end{aligned}
$$

and we estimate the three terms on the right-hand side of (50) separately.

Using (37) and the fact that $\Pi_{e}^{0} \llbracket \mathfrak{n} \times\left(\Pi_{h} z\right) \rrbracket\left(\right.$ respectively $\left.\Pi_{e}^{0} \llbracket \mathfrak{n} \cdot\left(\Pi_{h} z\right) \rrbracket\right)$ vanishes for all $e \in \mathcal{E}_{h}$ (respectively $e \in \mathcal{E}_{h}^{i}$ ), we rewrite the first term (following the notation in (38)) as

$$
\begin{aligned}
a_{\mathrm{h}}\left(\mathbf{u}-\mathbf{u}_{\mathrm{h}}, \boldsymbol{z}\right)= & a_{\mathrm{h}}\left(\mathbf{u}-\mathbf{u}_{\mathrm{h}}, \boldsymbol{z}-\Pi_{\mathrm{h}} \boldsymbol{z}\right)+\mathrm{a}_{\mathrm{h}}\left(\mathbf{u}-\mathbf{u}_{\mathrm{h}}, \Pi_{\mathrm{h}} \boldsymbol{z}\right) \\
= & a_{\mathrm{h}}\left(\mathbf{u}-\mathbf{u}_{\mathrm{h}}, \boldsymbol{z}-\Pi_{\mathrm{h}} \boldsymbol{z}\right) \\
& +\sum_{\mathrm{e} \in \mathcal{E}_{\mathrm{h}}} \int_{e}\left(\nabla \times \mathbf{u}-(\widehat{\nabla \times \mathbf{u}})_{\mathrm{T}_{e}}\right) \llbracket \mathfrak{n} \times\left(\Pi_{\mathrm{h}} \boldsymbol{z}\right) \rrbracket \mathrm{d} s
\end{aligned}
$$




$$
+\sum_{\mathfrak{e} \in \mathcal{E}_{\mathrm{h}}^{i}} \int_{e} \gamma\left(\nabla \cdot \mathbf{u}-(\widehat{\nabla \cdot \mathfrak{u}})_{\mathrm{T}_{e}}\right) \llbracket \mathfrak{n} \cdot\left(\Pi_{\mathrm{h}} z\right) \rrbracket \mathrm{d} s,
$$

from which we obtain the following estimate using (3), and Lemmas 8 and 10:

$$
a_{h}\left(\mathbf{u}-\mathbf{u}_{\mathrm{h}}, \boldsymbol{z}\right) \leqslant \mathrm{C}_{\epsilon}\left(\mathrm{h}^{2-\epsilon}\|\mathbf{f}\|_{\mathrm{L}_{2}(\Omega)}+\mathrm{h}^{1-\epsilon}\left\|\mathbf{u}-\mathbf{u}_{\mathrm{h}}\right\|_{\mathrm{h}}\right)\left\|\mathbf{u}-\mathbf{u}_{\mathrm{h}}\right\|_{\mathrm{L}_{2}(\Omega)} .
$$

Brenner et al. [15, pp. 526-527] gave details where an identical estimate is derived.

We now consider the second term on the right-hand side of (50). First

$$
\begin{aligned}
\sum_{\boldsymbol{e} \in \mathcal{E}_{\mathrm{h}}} \int_{e} \llbracket \mathfrak{n} \times \mathbf{u}_{\mathrm{h}} \rrbracket(\nabla \times \boldsymbol{z}) \mathrm{ds}= & \sum_{e \in \mathcal{E}_{\mathrm{h}}} \int_{e} \llbracket \mathfrak{n} \times \mathbf{u}_{\mathrm{h}} \rrbracket\left(\nabla \times \boldsymbol{z}-(\widehat{\nabla \times \boldsymbol{z}})_{\mathrm{T}_{e}}\right) \mathrm{ds} \\
& +\sum_{e \in \mathcal{E}_{\mathrm{h}}} \int_{e}\left(\Pi_{e}^{0} \llbracket \mathfrak{n} \times \mathbf{u}_{\mathrm{h}} \rrbracket\right)(\widehat{\nabla \times \boldsymbol{z}})_{\mathrm{T}_{e}} \mathrm{ds}
\end{aligned}
$$

where $(\widehat{\nabla \times \boldsymbol{z}})_{\mathrm{T}_{e}}$ is the mean of $\nabla \times \boldsymbol{z}$ on one of the triangles $\mathcal{T}_{e} \in \mathcal{T}_{\mathrm{h}}$ that has $\boldsymbol{e}$ as an edge.

The estimate below follows from (25), Lemma 10, (47) and the CauchySchwarz inequality:

$$
\begin{aligned}
& \sum_{\boldsymbol{e} \in \mathcal{E}_{\mathrm{h}}} \int_{e} \llbracket \mathfrak{n} \times \mathbf{u}_{\mathrm{h}} \rrbracket\left(\nabla \times \boldsymbol{z}-(\widehat{\nabla \times \boldsymbol{z}})_{\mathrm{T}_{e}}\right) \mathrm{ds} \\
& \leqslant \mathrm{Ch}\left\|\mathbf{u}-\mathbf{u}_{\mathrm{h}}\right\|_{\mathrm{L}_{2}(\Omega)}\left\|\mathbf{u}-\mathbf{u}_{\mathrm{h}}\right\|_{\mathrm{h}} .
\end{aligned}
$$

On the other hand, as in the derivation of (40), we obtain by the CauchySchwarz inequality, (25), (41) and (47),

$$
\begin{aligned}
& \sum_{\boldsymbol{e} \in \mathcal{E}_{\mathrm{h}}} \int_{e}\left(\Pi_{e}^{0} \llbracket \mathfrak{n} \times \mathbf{u}_{\mathrm{h}} \rrbracket\right)(\widehat{\nabla \times \boldsymbol{z}})_{\mathrm{T}_{e}} \mathrm{ds} \\
& =\sum_{\boldsymbol{e} \in \mathcal{E}_{\mathrm{h}}} \int_{e}\left(\Pi_{e}^{0} \llbracket \mathbf{n} \times\left(\mathbf{u}_{\mathrm{h}}-\mathbf{u}\right) \rrbracket\right)(\widehat{\nabla \times \boldsymbol{z}})_{\mathrm{T}_{e}} \mathrm{ds}
\end{aligned}
$$




$$
\begin{aligned}
& \leqslant \mathrm{Ch}\left\|\mathbf{u}-\mathbf{u}_{\mathrm{h}}\right\|_{\mathrm{h}}\|\nabla \times \boldsymbol{z}\|_{\mathrm{L}_{2}(\Omega)} \\
& \leqslant \mathrm{Ch}\left\|\mathbf{u}-\mathbf{u}_{\mathrm{h}}\right\|_{\mathrm{h}}\left\|\mathbf{u}-\mathbf{u}_{\mathrm{h}}\right\|_{\mathrm{L}_{2}(\Omega)} .
\end{aligned}
$$

Combining (52)-(54), we obtain

$$
\sum_{e \in \mathcal{E}_{\mathrm{h}}} \int_{e} \llbracket \mathfrak{n} \times \mathbf{u}_{\mathrm{h}}\|(\nabla \times \boldsymbol{z}) \mathrm{ds} \leqslant \mathrm{Ch}\| \mathbf{u}-\mathbf{u}_{\mathrm{h}}\left\|_{\mathrm{L}_{2}(\Omega)}\right\| \mathbf{u}-\mathbf{u}_{\mathrm{h}} \|_{\mathrm{h}} .
$$

Similarly, we have the following bound on the third term on the right-hand side of (50):

$$
\sum_{e \in \mathcal{E}_{h}^{i}} \int_{e} \gamma \llbracket \mathfrak{n} \cdot \mathbf{u}_{\mathrm{h}} \rrbracket(\nabla \cdot \boldsymbol{z}) \mathrm{ds} \leqslant \mathrm{Ch}\left\|\mathbf{u}-\mathbf{u}_{\mathrm{h}}\right\|_{\mathrm{L}_{2}(\Omega)}\left\|\mathbf{u}-\mathbf{u}_{\mathrm{h}}\right\|_{\mathrm{h}} .
$$

The estimate (44) follows from (50), (51), (55) and (56).

In the case where $\alpha>0$, the following theorem is an immediate consequence of Lemmas 5, 9, 11 and 12.

Theorem 13 Let $\alpha$ be positive. The following two discretization error estimates hold for the solution $\mathbf{u}_{\mathrm{h}}$ of (14):

$$
\begin{aligned}
& \left\|\mathbf{u}-\mathbf{u}_{\mathrm{h}}\right\|_{\mathrm{h}} \leqslant \mathrm{C}_{\epsilon} \mathrm{h}^{1-\epsilon}\|\mathbf{f}\|_{\mathrm{L}_{2}(\Omega)} \quad \text { for any } \epsilon>0 ; \\
& \left\|\mathbf{u}-\mathbf{u}_{\mathrm{h}}\right\|_{\mathrm{L}_{2}(\Omega)} \leqslant \mathrm{C}_{\epsilon} \mathrm{h}^{2-\epsilon}\|\mathbf{f}\|_{\mathrm{L}_{2}(\Omega)} \text { for any } \epsilon>0 \text {. }
\end{aligned}
$$

In the case where $\alpha \leqslant 0$, we have the following convergence theorem for the scheme (14), whose proof is based on Lemmas 6, 9, 11 and 12, and the approach of Schatz for indefinite problems [32]. The arguments are identical to those of an earlier proof [16, Theorem 4.5].

Theorem 14 Assume $-\alpha \geqslant 0$ is not one of the eigenvalues $\lambda_{\gamma, j}$ defined by (2). There exists a positive number $\mathrm{h}_{*}$ such that the discrete problem (14) is uniquely solvable for all $\mathrm{h} \leqslant \mathrm{h}_{*}$, in which case the following two discretization error estimates are valid:

$$
\left\|\mathbf{u}-\mathbf{u}_{\mathrm{h}}\right\|_{\mathrm{h}} \leqslant \mathrm{C}_{\epsilon} \mathrm{h}^{1-\epsilon}\|\mathbf{f}\|_{\mathrm{L}_{2}(\Omega)} \quad \text { for any } \epsilon>0 ;
$$




$$
\left\|\mathbf{u}-\mathbf{u}_{\mathrm{h}}\right\|_{\mathrm{L}_{2}(\Omega)} \leqslant \mathrm{C}_{\epsilon} \mathrm{h}^{2-\epsilon}\|\mathbf{f}\|_{\mathrm{L}_{2}(\Omega)} \quad \text { for any } \epsilon>0 .
$$

\section{$5 \quad$ Nonconforming meshes}

For simplicity we developed and analyzed the interior penalty method for triangulations (conforming meshes) of $\Omega$. But of course one of the main reasons for using an interior penalty method is that it can be applied to partitions with hanging nodes (nonconforming meshes). Here we indicate briefly how the scheme and results extend with minor modifications to such meshes.

Let $\mathcal{P}_{h}$ be a partition of $\Omega$ with hanging nodes satisfying the following condition: whenever a closed edge of a triangle in $\mathcal{P}_{h}$ contains a hanging node, then it is the union of closed edges of triangles in $\mathcal{T}_{h}$. An example of such a partition is depicted in Figure 2. For such a partition, we modify the definition of $\mathcal{E}_{h}$ as follows. Let $e$ be an (open) edge of a triangle in $\mathcal{P}_{h}$. Then $e \in \mathcal{E}_{\mathrm{h}}$ if and only if (i) it contains at least one hanging node, (ii) it is the common edge of two triangles in $\mathcal{P}_{h}$, that is, its endpoints are the common vertices of these triangles, or (iii) it is a subset of $\partial \Omega$. For example, the edge of the largest triangle (the diagonal of the square) in Figure 2 belongs to $\mathcal{E}_{\mathrm{h}}$ while the three edges on the diagonal from the three triangles on the other side do not. All together there are 12 edges in $\mathcal{E}_{\mathrm{h}}$ for the partition in Figure 2.

All the definitions in Section 3 can be extended to $\mathcal{P}_{h}$ in a straightforward fashion. For example, if $e \in \mathcal{E}_{h}^{i}$ has at least one hanging node, then $e$ is the edge of a triangle $T_{-} \in \mathcal{P}_{h}$ and also the union of edges $e_{1}, \ldots, e_{m}$ of the triangles $T_{+, 1}, \ldots, T_{+, m}$ in $\mathcal{P}_{h}$ that are on the other side of $e$ (compare with Figure 3 where $m=4$ ). We define, on $e$,

$$
\llbracket \mathfrak{n} \times \boldsymbol{v} \rrbracket=\left.\left(\boldsymbol{n}_{-} \times \boldsymbol{v}_{\mathrm{T}_{-}}\right)\right|_{e}+\left.\sum_{j=1}^{m}\left(\boldsymbol{n}_{+} \times \boldsymbol{v}_{\mathrm{T}_{+, j}}\right)\right|_{e_{j}},
$$




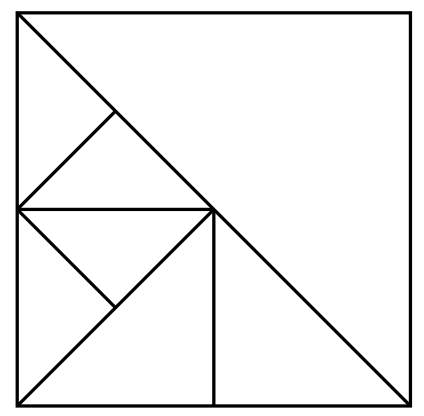

FiguRE 2: A triangular mesh with hanging nodes.

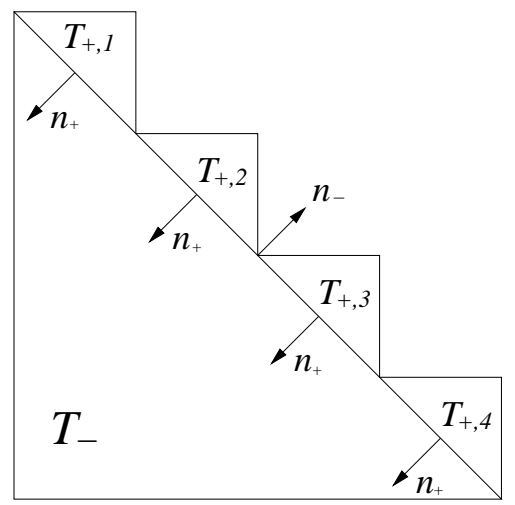

FiguRE 3: A triangular mesh with hanging nodes.

$$
\llbracket \mathfrak{n} \cdot \boldsymbol{v} \rrbracket=\left.\left(\mathbf{n}_{-} \cdot \boldsymbol{v}_{\mathrm{T}_{-}}\right)\right|_{e}+\left.\sum_{j=1}^{\mathrm{m}}\left(\mathbf{n}_{+} \cdot \boldsymbol{v}_{\mathrm{T}_{+, j}}\right)\right|_{e_{\mathrm{j}}} .
$$

The analysis in Section 4 remains valid for the type of nonconforming meshes under consideration because the crucial relations $\Pi_{e}^{0} \llbracket \mathfrak{n} \times\left(\mathbf{u}-\Pi_{\mathrm{h}} \mathbf{u}\right) \rrbracket=0$ for all $e \in \mathcal{E}_{h}$ and $\Pi_{e}^{0} \llbracket \mathbf{n} \cdot\left(\mathbf{u}-\Pi_{h} \mathbf{u}\right) \rrbracket=0$ for all $e \in \mathcal{E}_{h}^{i}$ hold for the modified definition of $\mathcal{E}_{h}$. Furthermore, all the estimates for $\mathbf{u}-\Pi_{h} \mathbf{u}$ can be carried out triangle by triangle and hence also hold for partitions with hanging nodes. 
Of course, the constants in the estimates now depend on the shape regularity of the partition, which roughly speaking involves the shape regularity of the triangles in $\mathcal{P}_{h}$ and the distribution of the hanging nodes on the edges in $\mathcal{E}_{h}$. Brenner $[12,13]$ detailed the concept of shape regularity of partitions.

\section{$6 \quad$ Numerical experiments}

In this section we report the results of a series of numerical experiments that corroborate our theoretical results. Both the $\mathrm{L}_{2}$ error $\left\|\mathbf{u}-\mathbf{u}_{\mathrm{h}}\right\|_{\mathrm{L}_{2}(\Omega)}$ and the energy error $\left\|\mathbf{u}-\mathbf{u}_{h}\right\|_{h}$ are computed for $\gamma=1$ in all the experiments.

In the first experiment we examine the convergence behavior of our numerical scheme on the square domain $(0,1)^{2}$ with conforming uniform meshes (Figure 4, left), where the exact solution is

$$
\mathbf{u}=\left[\begin{array}{l}
y(1-y) \\
x(1-x)
\end{array}\right]
$$

Table 1 gives the results for $\alpha=1,0$ and -1 . They show that the scheme (14) is second order accurate in the $\mathrm{L}_{2}$ norm and first order accurate in the energy norm, which agrees with the error estimates in Theorems 13 and 14.

In the second experiment we check the behavior of the scheme (14) on the square $(0,1)^{2}$ using nonconforming meshes with hanging nodes depicted in Figure 4 (right). The results in Table 2 show that the scheme also behaves as predicted in Theorems 13 and 14 .

The goal of the final experiment is to demonstrate the convergence behavior of our scheme on the L-shaped domain $(-0.5,0.5)^{2} \backslash[0,0.5]^{2}$. The right-hand side function is chosen to be

$$
\mathbf{f}=\left[\begin{array}{l}
1 \\
1
\end{array}\right] .
$$

The meshes are graded around the re-entrant corner $(0,0)$ using the refinement procedure of Bacuta et al. [7] with the grading parameter 1/3. The 
TABLE 1: Errors of the scheme on the square $(0,1)^{2}$ with conforming uniform meshes and exact solution given by (57).

\begin{tabular}{|ccccc|}
\hline$h$ & $\frac{\left\|\mathfrak{u}-\mathfrak{u}_{h}\right\|_{L_{2}(\Omega)}}{\|\mathfrak{u}\|_{\mathrm{L}_{2}(\Omega)}}$ & order & $\frac{\left\|\mathfrak{u}-\mathbf{u}_{h}\right\|_{h}}{\|\mathfrak{u}\|_{\mathrm{h}}}$ & order \\
\hline \multicolumn{5}{c}{$\alpha=1$} \\
\hline $1 / 8$ & $1.34 \mathrm{E}-01$ & 1.96 & $3.62 \mathrm{E}-01$ & 1.00 \\
$1 / 16$ & $3.29 \mathrm{E}-02$ & 2.03 & $1.79 \mathrm{E}-01$ & 1.02 \\
$1 / 32$ & $8.07 \mathrm{E}-03$ & 2.03 & $8.82 \mathrm{E}-02$ & 1.02 \\
$1 / 64$ & $1.99 \mathrm{E}-03$ & 2.02 & $4.38 \mathrm{E}-02$ & 1.01 \\
\hline \multicolumn{5}{c}{$\alpha=0$} \\
\hline $1 / 8$ & $1.49 \mathrm{E}-01$ & 2.01 & $3.83 \mathrm{E}-01$ & 1.03 \\
$1 / 16$ & $3.63 \mathrm{E}-02$ & 2.04 & $1.88 \mathrm{E}-01$ & 1.03 \\
$1 / 32$ & $8.88 \mathrm{E}-03$ & 2.03 & $9.25 \mathrm{E}-02$ & 1.02 \\
$1 / 64$ & $2.19 \mathrm{E}-03$ & 2.02 & $4.59 \mathrm{E}-02$ & 1.01 \\
\hline \multicolumn{5}{c}{$\alpha=-1$} \\
\hline $1 / 8$ & $1.69 \mathrm{E}-01$ & 2.08 & $4.07 \mathrm{E}-01$ & 1.07 \\
$1 / 16$ & $4.05 \mathrm{E}-02$ & 2.06 & $1.98 \mathrm{E}-01$ & 1.04 \\
$1 / 32$ & $9.88 \mathrm{E}-03$ & 2.03 & $9.76 \mathrm{E}-02$ & 1.02 \\
$1 / 64$ & $2.44 \mathrm{E}-03$ & 2.02 & $4.84 \mathrm{E}-02$ & 1.01 \\
\hline
\end{tabular}
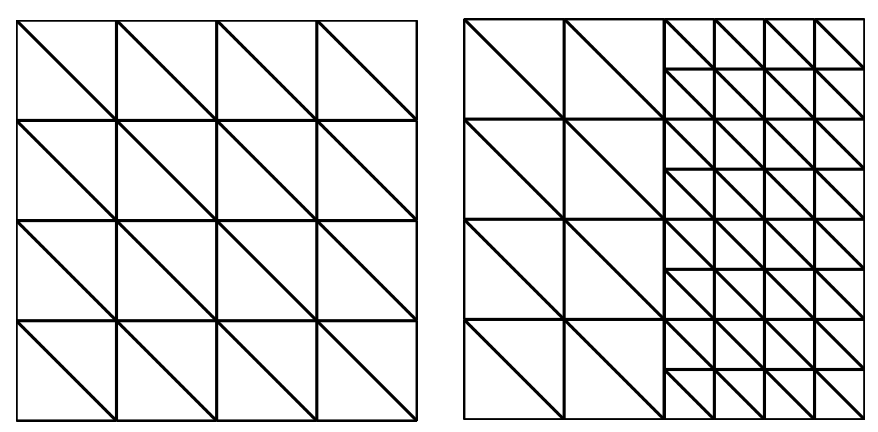

FiguRE 4: Conforming uniform mesh (left) and nonconforming mesh (right) on the square domain. 
TABLE 2: Errors of the scheme on the square $(0,1)^{2}$ with nonconforming meshes and exact solution given by (57).

\begin{tabular}{|ccccc|}
\hline $\mathrm{h}$ & $\frac{\left\|\mathfrak{u}-\mathbf{u}_{h}\right\|_{\mathrm{L}_{2}(\Omega)}}{\|\mathfrak{u}\|_{\mathrm{L}_{2}(\Omega)}}$ & order & $\frac{\left\|\mathfrak{u}-\mathfrak{u}_{h}\right\|_{\mathrm{h}}}{\|\mathfrak{u}\|_{\mathrm{h}}}$ & order \\
\hline \multicolumn{5}{c}{$\alpha=1$} \\
\hline $1 / 8$ & $8.82 \mathrm{E}-02$ & 1.80 & $2.98 \mathrm{E}-01$ & 0.90 \\
$1 / 16$ & $2.27 \mathrm{E}-02$ & 1.96 & $1.51 \mathrm{E}-01$ & 0.98 \\
$1 / 32$ & $5.69 \mathrm{E}-03$ & 2.00 & $7.59 \mathrm{E}-02$ & 1.00 \\
$1 / 64$ & $1.42 \mathrm{E}-03$ & 2.00 & $3.81 \mathrm{E}-02$ & 0.99 \\
\hline \multicolumn{5}{c}{$\alpha=0$} \\
\hline $1 / 8$ & $1.28 \mathrm{E}-01$ & 1.93 & $3.59 \mathrm{E}-01$ & 0.97 \\
$1 / 16$ & $3.21 \mathrm{E}-02$ & 2.00 & $1.80 \mathrm{E}-01$ & 1.00 \\
$1 / 32$ & $8.00 \mathrm{E}-03$ & 2.00 & $8.99 \mathrm{E}-02$ & 1.00 \\
$1 / 64$ & $1.96 \mathrm{E}-03$ & 2.03 & $4.03 \mathrm{E}-02$ & 1.15 \\
\hline \multicolumn{5}{c}{$\alpha=-1$} \\
\hline $1 / 8$ & $2.36 \mathrm{E}-01$ & 2.38 & $4.85 \mathrm{E}-01$ & 1.20 \\
$1 / 16$ & $5.52 \mathrm{E}-02$ & 2.10 & $2.35 \mathrm{E}-01$ & 1.01 \\
$1 / 32$ & $1.35 \mathrm{E}-02$ & 2.03 & $1.16 \mathrm{E}-01$ & 1.01 \\
$1 / 64$ & $3.31 \mathrm{E}-03$ & 2.03 & $5.80 \mathrm{E}-02$ & 1.00 \\
\hline \multicolumn{5}{c}{} \\
\hline
\end{tabular}



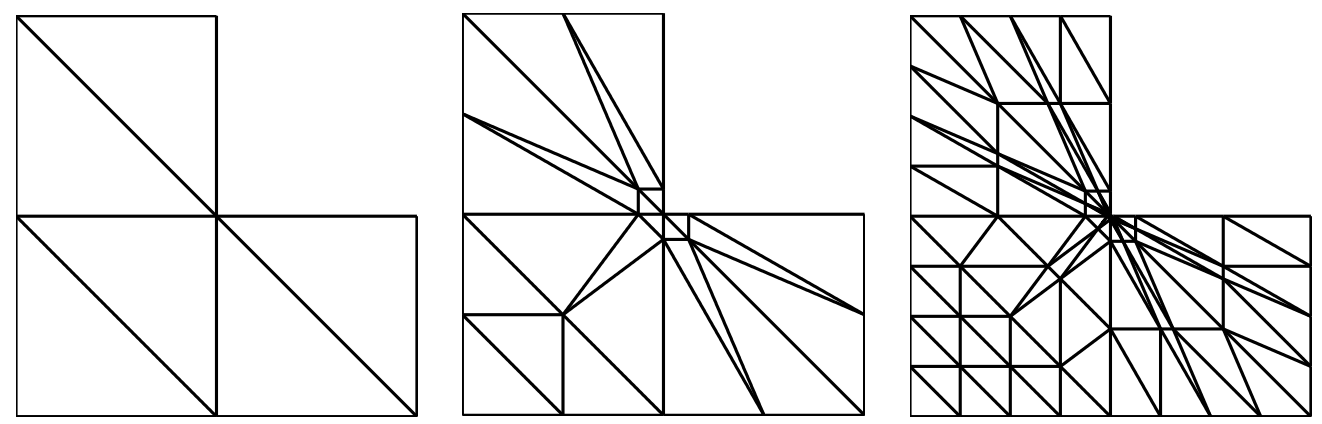

Figure 5: Graded meshes on the L-shaped domain.

first three levels of graded meshes are depicted in Figure 5. The results in Table 3 demonstrate that the scheme is second order accurate in the $\mathrm{L}_{2}$ norm and first order accurate in the energy norm.

\section{Concluding remarks}

We extended the nonconforming method of our earlier work [15] to an interior penalty method that can be applied to nonconforming meshes. This interior penalty method enjoys optimal convergence rates without any tuning of penalty parameters.

The condition numbers of the discrete problems are worsened by the weak over penalization. It is therefore important to have good preconditioners in the case of fine meshes. For the Laplace operator, efficient preconditioners for interior penalty methods with weak over penalisation were developed by Owens et al. [31, 19]. The development of good preconditioners for the family of $\mathrm{H}(\operatorname{curl} ; \Omega) \cap \mathrm{H}(\operatorname{div} ; \Omega)$ methods in earlier work $[16,15,17,20,18]$ and this article is currently under investigation. 
TABLE 3: Errors of the scheme on the L-shaped domain with graded meshes and right-hand side given by (58).

\begin{tabular}{|crrrc|}
\hline $\mathrm{h}$ & $\frac{\left\|\mathfrak{u}-\mathfrak{u}_{h}\right\|_{\mathrm{L}_{2}(\Omega)}}{\|\mathfrak{u}\|_{\mathrm{L}_{2}(\Omega)}}$ & order & $\frac{\left\|\mathfrak{u}-\mathfrak{u}_{h}\right\|_{\mathrm{h}}}{\|\mathfrak{u}\|_{h}}$ & order \\
\hline \multicolumn{5}{c}{$\alpha=1$} \\
\hline $1 / 16$ & $4.77 \mathrm{E}-01$ & 1.67 & $1.02 \mathrm{E}+00$ & 1.13 \\
$1 / 32$ & $1.28 \mathrm{E}-01$ & 1.89 & $4.65 \mathrm{E}-01$ & 1.13 \\
$1 / 64$ & $3.23 \mathrm{E}-02$ & 1.99 & $2.20 \mathrm{E}-01$ & 1.08 \\
$1 / 128$ & $8.03 \mathrm{E}-03$ & 2.01 & $1.07 \mathrm{E}-01$ & 1.04 \\
\hline \multicolumn{5}{c}{$\alpha=0$} \\
\hline $1 / 16$ & $6.21 \mathrm{E}-01$ & 2.11 & $1.14 \mathrm{E}+00$ & 1.37 \\
$1 / 32$ & $1.52 \mathrm{E}-01$ & 2.03 & $5.01 \mathrm{E}-01$ & 1.19 \\
$1 / 64$ & $3.74 \mathrm{E}-02$ & 2.02 & $2.34 \mathrm{E}-01$ & 1.10 \\
$1 / 128$ & $9.22 \mathrm{E}-03$ & 2.02 & $1.13 \mathrm{E}-01$ & 1.05 \\
\hline \multicolumn{5}{c}{$\alpha=-1$} \\
\hline $1 / 16$ & $9.07 \mathrm{E}-01$ & 3.45 & $1.46 \mathrm{E}+00$ & 1.48 \\
$1 / 32$ & $1.90 \mathrm{E}-01$ & 2.26 & $5.47 \mathrm{E}-01$ & 1.37 \\
$1 / 64$ & $4.49 \mathrm{E}-02$ & 2.08 & $2.55 \mathrm{E}-01$ & 1.15 \\
$1 / 128$ & $1.10 \mathrm{E}-02$ & 2.04 & $1.22 \mathrm{E}-01$ & 1.06 \\
\hline
\end{tabular}


Acknowledgements The work in this article is supported in part by the National Science Foundation under Grant No. DMS-07-13835.

\section{References}

[1] Th. Apel. Anisotropic Finite Elements. Teubner, Stuttgart, 1999. C952

[2] Th. Apel, A.-M. Sändig, and J.R. Whiteman. Graded mesh refinement and error estimates for finite element solutions of elliptic boundary value problems in non-smooth domains. Math. Methods Appl. Sci., 19:63-85, 1996. C952

[3] F. Assous, P. Ciarlet, Jr., S. Labrunie, and J. Segré. Numerical solution to the time-dependent Maxwell equations in axisymmetric singular domains: the singular complement method. J. Comput. Phys., 191:147-176, 2003. C948

[4] F. Assous, P. Ciarlet, Jr., E. Garcia, and J. Segré. Time-dependent Maxwell's equations with charges in singular geometries. Comput. Methods Appl. Mech. Engrg., 196:665-681, 2006. C948

[5] F. Assous, P. Ciarlet Jr., S. Labrunie, and S. Lohrengel. The singular complement method. In N. Debit, M. Garbey, R. Hoppe, D. Keyes, Y. Kuznetsov, and J. Périaux, editors, Domain Decomposition Methods in Science and Engineering, pages 161-189. CIMNE, Barcelona, 2002. C948

[6] F. Assous, P. Ciarlet, Jr., and E. Sonnendrücker. Resolution of the Maxwell equation in a domain with reentrant corners. M2AN Math. Model. Numer. Anal., 32:359-389, 1998. C950

[7] C. Băcuţă, V. Nistor, and L. T. Zikatanov. Improving the rate of convergence of 'high order finite elements' on polygons and domains with cusps. Numer. Math., 100:165-184, 2005. C952, C967 
[8] K. J. Bathe, C. Nitikitpaiboon, and X. Wang. A mixed displacement-based finite element formulation for acoustic fluid-structure interaction. Comput. Struct., 56:225-237, 1995. C948

[9] A. Bermúdez and R. Rodríguez. Finite element computation of the vibration modes of a fluid-solid system. Comput. Methods Appl. Mech. Engrg., 119:355-370, 1994. C948

[10] D. Boffi and L. Gastaldi. On the "-grad div $+s$ curl rot" operator. In Computational fluid and solid mechanics, Vol. 1, 2 (Cambridge, MA, 2001), pages 1526-1529. Elsevier, Amsterdam, 2001. C948

[11] A.-S. Bonnet-Ben Dhia, C. Hazard, and S. Lohrengel. A singular field method for the solution of Maxwell's equations in polyhedral domains. SIAM J. Appl. Math., 59:2028-2044, 1999. C948

[12] S. C. Brenner. Poincaré-Friedrichs inequalities for piecewise $\mathrm{H}^{1}$ functions. SIAM J. Numer. Anal., 41:306-324, 2003. C967

[13] S. C. Brenner. Korn's inequalities for piecewise $\mathrm{H}^{1}$ vector fields. Math. Comp., 73:1067-1087, 2004. C967

[14] S. C. Brenner and C. Carstensen. Finite Element Methods. In E. Stein, R. de Borst, and T.J.R. Hughes, editors, Encyclopedia of Computational Mechanics, pages 73-118. Wiley, Weinheim, 2004. C952

[15] S. C. Brenner, J. Cui, F. Li, and L.-Y. Sung. A nonconforming finite element method for a two-dimensional curl-curl and grad-div problem. Numer. Math., 109:509-533, 2008. C948, C949, C950, C954, C963, C970

[16] S. C. Brenner, F. Li, and L.-Y. Sung. A locally divergence-free nonconforming finite element method for the time-harmonic Maxwell equations. Math. Comp., 76:573-595, 2007. C949, C957, C958, C964, C970 
[17] S. C. Brenner, F. Li, and L.-Y. Sung. A nonconforming penalty method for a two dimensional curl-curl problem. M3AS, 19:651-668, 2009. C949, C970

[18] S. C. Brenner, F. Li, and L.-Y. Sung. A locally divergence-free interior penalty method for two dimensional curl-curl problems. SIAM J. Numer. Anal., 46:1190-1211, 2008. C949, C956, C970

[19] S. C. Brenner, L. Owens, and L.-Y. Sung. A weakly over-penalized symmetric interior penalty method. ETNA, 30:107-127, 2008. C970

[20] S. C. Brenner and L.-Y. Sung. A quadratic nonconforming element for $\mathrm{H}(\operatorname{curl} ; \Omega) \cap \mathrm{H}(\operatorname{div} ; \Omega)$. Appl. Math. Lett., 22:892-896, 2009. C949, C970

[21] P. Ciarlet, Jr. Augmented formulations for solving Maxwell equations. Comput. Methods Appl. Mech. Engrg., 194:559-586, 2005. C948

[22] M. Costabel. A coercive bilinear form for Maxwell's equations. $J$. Math. Anal. Appl., 157:527-541, 1991. C948

[23] M. Costabel and M. Dauge. Singularities of electromagnetic fields in polyhedral domains. Arch. Ration. Mech. Anal., 151:221-276, 2000. C950

[24] M. Costabel and M. Dauge. Weighted regularization of Maxwell equations in polyhedral domains. Numer. Math., 93:239-277, 2002. C948

[25] M. Costabel, M. Dauge, and C. Schwab. Exponential convergence of hp-FEM for Maxwell equations with weighted regularization in polygonal domains. Math. Models Methods Appl. Sci., 15:575-622, 2005. C948

[26] M. Crouzeix and P.-A. Raviart. Conforming and nonconforming finite element methods for solving the stationary Stokes equations I. RAIRO Anal. Numér., 7:33-75, 1973. C948, C954 
[27] M. A. Hamdi, Y. Ousset, and G. Verchery. A displacement method for the analysis of vibrations of coupled fluid-structure systems. Internat. J. Numer. Methods Engrg., 13:139-150, 1978. C948

[28] C. Hazard and S. Lohrengel. A singular field method for Maxwell's equations: Numerical aspects for 2D magnetostatics. SIAM J. Numer. Anal., 40:1021-1040, 2002. C948

[29] J.-M. Jin. The Finite Element Method in Electromagnetics (Second Edition). John Wiley \& Sons, Inc., New York, 2002. C948

[30] P. Monk. Finite Element Methods for Maxwell's Equations. Oxford University Press, New York, 2003. C948, C950, C954

[31] L. Owens. Multigrid Methods for Weakly Over-Penalized Interior Penalty Methods. PhD thesis, University of South Carolina, 2007. C970

[32] A. Schatz. An observation concerning Ritz-Galerkin methods with indefinite bilinear forms. Math. Comp., 28:959-962, 1974. C964

\section{Author addresses}

1. S. C. Brenner, Department of Mathematics and Center for Computation and Technology, Louisiana State University, Baton Rouge, LA 70803, USA.

mailto: brenner@math. Isu .edu

2. J. Cui, Department of Mathematics, Louisiana State University, Baton Rouge, LA 70803, USA.

3. L.-Y. Sung, Department of Mathematics, Louisiana State University, Baton Rouge, LA 70803, USA. 